Cahiers « Mondes anciens »

MONDES

ANCIENS

Histoire et anthropologie des mondes anciens

$6 \mid 2015$

Mères grecques

\title{
Les relations filles/mères autour de la question du mariage dans l'Athènes classique
}

Mothers-Daughters Relationships and the Issue of Marriage in Classical Athens

\section{Nathalie Ernoult}

\section{(2) OpenEdition}

Journals

Édition électronique

URL : http://journals.openedition.org/mondesanciens/1339

DOI : 10.4000/mondesanciens.1339

ISSN : 2107-0199

Éditeur

UMR 8210 Anthropologie et Histoire des Mondes Antiques

Référence électronique

Nathalie Ernoult, «Les relations filles/mères autour de la question du mariage dans l'Athènes

classique », Cahiers "Mondes anciens » [En ligne], 6 | 2015, mis en ligne le 16 février 2015, consulté le

30 avril 2019. URL : http://journals.openedition.org/mondesanciens/1339; DOI : 10.4000/

mondesanciens.1339

Ce document a été généré automatiquement le 30 avril 2019.

Les Cahiers "Mondes Anciens » sont mis à disposition selon les termes de la licence Creative Commons Attribution - Pas d'Utilisation Commerciale - Pas de Modification 4.0 International. 


\title{
Les relations filles/mères autour de la question du mariage dans l'Athènes classique
}

Mothers-Daughters Relationships and the Issue of Marriage in Classical Athens

\author{
Nathalie Ernoult
}

1 Nous ne savons malheureusement que très peu de choses sur les liens entre les mères et leurs filles dans l'Athènes classique ${ }^{1}$. Les sources grecques, peu loquaces, préfèrent s'attarder sur les relations entre les pères et leurs filles. En effet, c'est le père qui, indique Isée, dès la naissance de l'enfant, fille ou garçon, organise les rituels permettant d'intégrer le nouveau-né dans la maison familiale ${ }^{2}$; c'est le père qui, à l'issue de ces rituels, légitime et établit la filiation de l'enfant, lui donne un nom et une reconnaissance sociale $^{3}$. Tout acte public concernant les filles qui implique la cité, de la naissance à la mort $^{4}$ en passant par le mariage ${ }^{5}$, est accompli par le père ou à défaut par les hommes de la famille.

2 Si les mères transmettent à leurs fils statut et patrimoine qui vont faire d'eux des citoyens, il semblerait que ce qu'elles transmettent à leurs filles est du domaine strictement familiale et ne sort de l'oikos que lors de certaines fêtes religieuses. Dans l'idéal masculin des Athéniens de la bonne société de l'époque classique ${ }^{6}$ tel que nous l'a transmis Xénophon dans l'Économique, les mères apprennent surtout à leur filles à se soumettre à la volonté des hommes : « Ma mère m'a dit que mon affaire à moi c'est d'être sage (sôphronein) ${ }^{7}$. " Et il est fort probable que dans la bonne société athénienne, les mères enseignaient à leurs filles comment incarner le rôle social correspondant à l'idéal féminin grec, c'est-à-dire à se préparer à devenir une bonne épouse, une bonne mère qui met au monde un enfant légitime mâle et une femme socialisée qui assure les fonctions religieuses propres à son sexe.

3 Si les femmes sont exclues des institutions politiques, elles ont vraisemblablement un rôle déterminant au sein de l'oikos en transmettant, bien ou mal, à leurs filles les valeurs sociales qui vont définir leur statut au sein de la cité. Cette transmission de la mère à la 
fille commence sans doute, comme le suggère Yvette Morizot $^{8}$, dès la naissance de l'enfant, par l'accomplissement d'actes religieux. En effet, un relief en marbre trouvé en Thessalie met en scène un groupe de femmes, dont la mère, présentant un nouveau-né à Artémis, accompagnée d'un sacrifice en remerciement pour un accouchement réussi. Ce nourrisson est-il une fille ou un garçon? On ne le sait pas : le bébé est souvent représenté emmailloté. Mais il est plausible qu'un tel rite ait lieu quel que soit le sexe de l'enfant. Par ailleurs, il est tout à fait possible que la mère soit officieusement associée au père pour choisir le nom de son enfant, fille ou garçon lors de la cérémonie de la dekatêe. Dans certaines cités, le nom des filles se transmet de la grand-mère maternelle à la petite fille instituant ainsi une généalogie féminine ${ }^{10}$. Cette transmission se poursuit dans l'enfance et à l'adolescence par l'apprentissage des mythes, ces « contes de bonnes femmes » bannis par Platon comme autant de mensonges sur les dieux ${ }^{11}$ mais qui constituent le socle de la formation culturelle des jeunes filles. Les mères initient également leurs filles aux rites, danses et chants, nécessaires à leur participation aux fêtes solennelles que donne la cité en l'honneur des dieux ${ }^{12}$. Elles leurs enseignent également toutes les activités liées à la bonne gestion de l'oikos, la préparation des repas, le travail de la laine et la distribution des tâches domestiques aux esclaves. Ainsi, quand tout se passe bien, le savoir-faire des femmes fondé sur leur mémoire collective se transmet de mère en fille, d'une génération à l'autre. Mais il semblerait que, dans l'idéal, la transmission de la mère à la fille trouve son telos dans le mariage, au moment où cette dernière se sépare de sa mère pour intégrer un nouveau foyer.

\section{Le telos de la transmission mère/fille : le mariage}

4 Le mariage est, souligne Louise Bruit Zaidman, "l'accomplissement nécessaire et souhaité, qui donnera tout son sens à la vie de femme et de mère, pour laquelle est née la fille $^{13}$ ». Les mères sont-elles pour autant, comme l'affirment Lydie Bodiou, Pierre Brulé et Laurence Pierini, exclues « de cet instant qui fait de leur fille une femme ${ }^{14}$ »? Rien n'est moins sûr. Au contraire, tout porte à croire que la mère participe activement à toutes les étapes du mariage de sa fille. Certes, civilement, les mères n'ont aucun rôle dans l'enguê, cet acte par lequel le père donne sa fille à son futur époux. Mais le mariage, ce passage «traumatisant ${ }^{15}$ » qui transfère la jeune fille d'une maison à une autre, fait l'objet de nombreux rites préparatoires auxquels la mère est étroitement associée.

5 Nos informations sur le mariage sont fragmentaires et incomplètes ${ }^{16}$. Les orateurs attiques n'y font référence que pour défendre ou contester sa légitimité. S'ils évoquent les nombreux rites qui sanctionnent la légitimité de l'union aux yeux de la cité, le rôle de la mère n'est jamais présent dans le débat public. Il faut donc regarder du côté de la tragédie, plus particulièrement dans le théâtre d'Euripide, pour avoir un aperçu de l'intervention des mères dans les rituels du mariage. Le poète est assurément l'un des rares Athéniens à avoir donné aux femmes une parole que lui refuse la cité et une place égale à celle des hommes ${ }^{17}$. Et on va voir que lorsqu'il s'agit de marier leur fille, les mères tragiques revendiquent non seulement de participer activement aux préparatifs du mariage, de conduire leur enfant dans la demeure de son époux mais également de donner leur consentement à l'union.

6 Bien que le mariage soit toujours décidé par le père, il semble en effet que le gamos d'une fille nécessite l'accord tacite de sa mère qui l'accompagne dans ce changement de statut. Ce consentement est requis dès la première pièce du tragédien, Alceste. Alors que 
l'héroïne éponyme s'apprête à faire don de sa vie pour son époux Admète, celle-ci supplie ce dernier de ne pas introduire dans sa maison une seconde épouse, forcément hostile, et donc incapable de préparer dignement sa fille à devenir une femme : "Quelle femme trouveras-tu dans la compagne de ton père? Ah! Qu'elle n'aille pas, t'infligeant quelque honteux renom, dans la fleur de ta jeunesse, ruiner ton hymen! Tu n'auras pas de mère pour te donner en mariage (numpheusei) ni te réconforter dans tes couches, à l'heure où rien ne vaut l'affection maternelle ${ }^{18}$.»

7 Iphigénie à Aulis est certainement la pièce où est le mieux évoquée l'importance du rôle de la mère dans le mariage de sa fille. Alors qu'il s'apprête à la sacrifier, Agamemnon, dans Iphigénie à Aulis, fait venir Iphigénie sous le prétexte fallacieux de la marier à Achille. Ignorant ses intentions, Clytemnestre tout à la joie des préparatifs du mariage, accompagne sa fille : «Il était naturel qu'elle suivît sa fille pour la marier et donner ce qu'elle a de plus cher19. . Mais lorsqu'elle apprend l'intention du roi de marier seul leur fille, elle s'insurge : "Comment, sans la mère, accomplirez-vous ce qui me revient ?20 " Elle refuse alors, malgré l'ordre d'Agamemnon, de rentrer à Argos auprès de ses autres filles : "À toi d'aller régler les affaires du dehors. À moi celles de la maison et ce qui concerne fiancés ou jeunes filles ${ }^{21}$. » Achille, quant à lui, furieux d'avoir été piégé, accuse Agamemnon d'avoir utilisé son nom pour obtenir le consentement de Clytemnestre aux prétendues noces de sa fille: "Il aurait dû lui-même me demander mon nom comme piège pour son enfant. À moi plus que tout autre Clytemnestre eût consenti à donner ( ekdouna) sa fille pour époux ${ }^{22}$. " Ainsi, Clytemnestre n'entend pas laisser Agamemnon la dessaisir, concernant le mariage d'Iphigénie, de ses prérogatives de mère. Elle acceptera également le mariage malheureux d'Électre avec le paysan Pylade ${ }^{23}$.

Les affaires de l'oikos ainsi que le mariage sont le domaine des femmes et des mères s'exclame Clytemnestre. Et de fait, les mères tragiques ont un rôle prépondérant dans tous les rituels de la cérémonie nuptiale. Elles assistent leurs filles lors de la loutrophorie, leur apportent l'eau du bain nuptial ${ }^{24}$ et procèdent à leur toilette ${ }^{25}$. Le jour des noces, ce sont elles qui coiffent leur fille d'une couronne de myrte, gage d'une fécondité heureuse. Ainsi, Cassandre, après avoir invité sa mère à danser et chanter pour célébrer ses noces avec Agamemnon ${ }^{26}$, lui demande de poser sur son front la couronne nuptiale ${ }^{27}$. Clytemnestre croyant marier Iphigénie à Achille lui avait couronné la tête avant de la conduire auprès de ce dernier ${ }^{28}$. Elle envisage ensuite de donner un repas de noces, la thoinê, propre aux femmes, parallèlement à celui qu'est censé donner Agamemnon pour les hommes après les rites préliminaires des proteleia ${ }^{29}$. Et comme le veut l'usage ${ }^{30}$, c'est à la mère qu'incombe la charge de conduire, aux sons de l'hyménée, sa fille jusqu'à son époux $^{31}$. Ainsi, Clytemnestre, qualifiée pour l'occasion de numphagogos, exige, malgré l'opposition d'Agamemnon, de conduire le cortège et de porter la torche nuptiale lors de la procession - une torche que porte également la belle-mère de la mariée, lorsqu'elle accueille les jeunes époux devant la porte et les conduit à la chambre nuptiale ${ }^{32}$. C'est de ce rituel que Jocaste se plaint d'avoir été exclue lors du mariage de son fils Polynice ${ }^{33}$ et en son absence, comme le fait remarquer Créuse à son fils Ion, l'enfant à naître est un bâtard ${ }^{34}$.

9 Ainsi, le mariage est au centre de la relation mères/filles. Ce sont les mères qui préparent leurs filles à accomplir leur destin de filles de la cité, à quitter la douceur du domicile familial pour rejoindre celui de l'époux auquel elles seront désormais attachées. La séparation est douloureuse pour l'une comme pour l'autre mais, comme l'admet Clytemnestre, «c'est la loi de la vie ${ }^{35} »$. Et lorsque, comme Cassandre dans les Troyennes, 
les filles sont réticentes au mariage, elles supplient leur mère de les y conduire de force : «Mère, à toi de me conduire [aux épousailles]; et si tu vois en moi quelque répugnance, entraîne m'y de force ${ }^{36}$.»

Il faut certes, comme le souligne Claire Nancy, se garder de voir dans l'œuvre d'Euripide un reflet fiable des mentalités de ses contemporains ${ }^{37}$. Les mères et les filles des tragédies sont des héroïnes et leurs comportements se manifestent lors de situations exceptionnelles et spécifiques à chaque pièce. Cependant, comme l'observe Anne-Marie Vérilhac, ce théâtre joué devant le peuple lors des Grandes Dyonisies, rencontre forcément un écho dans les représentations mentales des Athéniens: "Ses héroïnes mythiques offrent une image des Athéniennes $d u v^{e}$ siècle assez reconnaissable pour que les spectateurs ne fassent pas le départ entre les personnages qu'ils viennent de voir sur la scène et les épouses, les filles ou les sœurs qu'ils retrouvent chez eux au retour du théâtre ${ }^{38}$.» Par ailleurs, on pense aujourd'hui qu'il est vraisemblable que les femmes assistaient aux représentations théâtrales et pouvaient ainsi s'identifier aux personnages tragiques féminins ${ }^{39}$. Et si l'on quitte l'imaginaire atypique de la tragédie pour celui plus prosaïque des épitaphes funéraires, on y voit la détresse des mères de jeunes filles mortes trop tôt, se plaignant de ne pas avoir complètement accompli leurs devoir envers elles, faute de les avoir conduites jusqu'à la couche nuptiale: "Tu es donc morte avant l'hymen, Philainion, et ta mère ne t'a pas conduite, l'heure venue, à la chambre d'un fiancé; mais les joues déchirées pitoyablement, à quatorze ans, elle t'a ensevelie dans cette tombe ${ }^{40}$. " Une mère qui, pour une raison ou pour une autre, n'a pas pu accompagner sa fille dans le passage de la parthenos à la numphê, ne peut voir l'aboutissement de son devoir de transmission. Une transmission qui, semble-t-il, ne concerne que les filles. En effet, dans son étude sur la poésie funéraire, Anne-Marie Vérilhac montre que les poètes se lamentent sur la mort prématurée des filles avant le mariage tandis que la mort prématurée des garçons est évoquée avant leur première barbe ${ }^{41}$.

\section{Déméter et Korè : une transmission partiellement compromise}

11 C'est cet échec du devoir de la mère envers sa fille que raconte, entre autres choses, le mythe transmis par l'Hymne homérique à Déméter daté vers la fin du $\mathrm{VII}^{\mathrm{e}}$ siècle ou le $\mathrm{VI}^{\mathrm{e}}$ siècle. Il témoigne en effet de la douleur et de la révolte d'une mère dépossédée du mariage de sa fille, laquelle, après avoir été donnée en mariage par son père, Zeus, à son oncle Hadès, a été enlevée de force par son époux ${ }^{42}$. Comme le remarque Louise Bruit Zaidman : "Déméter est la mère par excellence, mère d'une fille, Korê, qui devient le paradigme des filles offertes au mariage à travers la violence du rapt et la rupture brutale de la vie de jeune fille auprès de la mère ${ }^{43}$. » Ce rapt est particulièrement violent dans la mesure où il a lieu, d'une part à l'insu de la mère qui part à la recherche de sa fille ne sachant ce qui lui est advenu, et d'autre part, en dépit de la résistance de la fille qui, emmenée aux Enfers, implore un père sourd à ses supplications.

Ayant appris la vérité par Hélios, Déméter, mortifiée par l'acte de son frère et époux, se révolte et quitte l'olympe "pour aller vers les cités des hommes et leurs grasses cultures" (v. 92-93). Mais ce séjour sur terre ne calme pas la colère de la déesse de l'agriculture et des moissons. Pour se venger, elle empêche la terre de produire ses fruits 
en cachant le grain, privant du même coup les dieux des timai offertes par les hommes. Inquiets de l'extinction de la race des mortels par la famine, les dieux tentent de persuader Déméter de revenir sur l'olympe. La déesse n'y consent qu'à la condition de revoir sa fille. Zeus envoie alors Hermès au royaume d'Hadès chercher Perséphone ${ }^{44}$ pour la ramener auprès de sa mère. Avant de consentir à laisser partir son épouse, Hadès lui donne à manger de la nourriture des Enfers, soit des pépins de grenade, symbole de fécondité et du mariage ${ }^{45}$, afin d'éviter que Perséphone ne reste définitivement auprès de sa mère. Les dieux trouvent alors un compromis : Perséphone passera un tiers du temps aux Enfers auprès de son époux et les deux tiers restants auprès de sa mère. Certes, le mythe relate, ainsi que le rappelle Louise Bruit Zaidman, une «rupture nécessaire aboutissant au mariage marquant dans un premier temps la séparation de la mère et de la fille avant leurs retrouvailles dans le culte qui célèbre la fécondité des champs et des cités ${ }^{46}$ ". Mais au-delà de cette "rupture nécessaire ", il raconte également la douleur et le combat d'une mère face à la violence de cette séparation imposée par son époux et son gendre. Et dans cet affrontement avec Zeus, Déméter n'a pas d'autre moyen que de lutter avec les armes qui lui sont propres ${ }^{47}$, celles de donner vie ou non aux semences nourricières. Il n'y a ni gagnant ni perdant dans ce conflit entre le père et la mère résolu par un compromis qui est une entorse à la norme puisque, comme le remarque Claude Calame, « Perséphone ne connaît ni le détachement définitif de la demeure de son père et de son statut de vierge, ni l'agrégation entière à la maison et à l'autorité du mari à qui elle doit donner une descendance ${ }^{48} »$. Perséphone n'est donc ni tout à fait une jeune fille, ni tout à fait une femme adulte ou, plus exactement, elle est alternativement l'une et l'autre 49.

Sans revenir sur les différentes interprétations de l'hymne homérique à Déméter ${ }^{50}$ - dont j'ai fait un exposé très incomplet pour ne m'en tenir qu'aux relations entre le père, la mère, la fille et son époux -, ne peut-on pas voir dans ce mythe, parmi bien d'autres choses, une mise en cause de la toute puissance du père ? Effectivement, Zeus donne Koré en mariage à Hadès sans en informer Déméter. Ainsi, le père s'est arrogé le droit, sans consulter la mère et donc sans son accord, de donner sa fille en mariage. Mise devant ce fait accompli, la mère, n'a pas pu, comme c'est l'usage la conduire vers la demeure de son époux. Il est vrai qu'on a du mal à imaginer que Déméter, ni d'ailleurs aucune autre mère, eût consenti à donner sa fille en mariage au dieu des Enfers. Mais finalement, le compromis entre Zeus, Déméter et Hadès est une victoire partielle de la mère et la fille contre la décision du père. Perséphone est certes l'épouse d'Hadès, le mariage n'est pas remis en cause, néanmoins ce mariage est incomplet puisqu'il n'a qu'une durée limitée dans l'année, Perséphone quittant le reste du temps son époux pour retrouver sa mère. La mère reprend alors le pouvoir sur le père. Il est vrai, cependant, que la mère Déméter est une déesse et, comme le rappelle Nicole Loraux, une « déesse n'est pas une femme ${ }^{51}$ ».

\section{Les relations filles/mères vues par les orateurs attiques}

Le personnage de la mère est assez présent dans la prose des orateurs d'époque classique, principalement dans les plaidoyers civils, notamment ceux qui traitent de conflits familiaux et de captation d'héritage. En effet, depuis le décret de Périclès de 451 av. J.-C. restreignant le droit à la citoyenneté aux enfants nés de père citoyen et de mère fille de citoyen, la mère joue un rôle essentiel dans la transmission du statut et des biens ${ }^{52}$. C'est 
elle qui, pour reprendre les termes de Claude Vial, « permet la continuation de la lignée ; qui permet la légitimité des enfants; qui permet d'hériter, de jouir des droits civiques, d'avoir des parents par le sang et par l'alliance ${ }^{53}$ ». Il s'agit dès lors pour le plaideur d'établir sa filiation maternelle ou de contester celle de son adversaire pour revendiquer la succession d'un parent. Les mères sont également présentes parce qu'elles ont une dot qui peut susciter bien des convoitises ${ }^{54}$. Par ailleurs, il arrive parfois qu'une mère, lorsqu'elle est fille épiclère, passe sous la tutelle de son fils, ce qui assigne à ce dernier, rappelle Démosthène, une obligation alimentaire envers sa mère, la gerotrophia: « $\mathrm{Si}$ l'épiclère a un fils et qu'il dépasse de deux ans l'âge de la puberté, ce fils receuillera le patrimoine à charge de verser les aliments à sa mère. Ainsi, au terme de la loi, les fils, après leur puberté, acquièrent la puissance sur leur mère (kurious tês mêtros), à charge de lui verser les aliments $s^{55}$. Il est d'ailleurs fréquent qu'une mère habite avec son fils ${ }^{56}$. Les filles, n'ayant pas de biens dont elles puissent disposer pour elles-mêmes et ayant peu l'occasion de vivre avec une vieille mère puisque leur destin est de quitter assez jeune l' oikos paternel, ne sont, quant à elles, probablement pas soumises à la gerotrophia.

Certains orateurs évoquent parfois la bienveillance d'une mère qui protège ses enfants et les secoure en l'absence de leur père ${ }^{57}$. Elle est celle qui "par nature, supporte plus patiemment que personne les injures de ses enfants, qui est si sensible aux moindres soin $^{58}$ ». Inversement, dans l'univers mouvementé et cruel dépeint par les orateurs attiques, il $\mathrm{y}$ a aussi de mauvaises mères qui élèvent mal leurs enfants ${ }^{59}$, ou ont une conduite qui déshonore la famille et la cité6 ${ }^{6}$. Il y a également de mauvais fils qui maltraitent leur mère ${ }^{61}$. En revanche, dans les plaidoyers on ne voit jamais de mauvaises filles brutalisant leur géniteurs.

16 Ainsi, qu'elle soit bonne ou mauvaise, la mère joue bien souvent un rôle décisif dans l'argumentation des plaideurs. Toutefois, celle-ci a rarement une identité propre; la plupart du temps, les orateurs ne convoquent leur mère, ou celle des autres, que pour les situer dans un système de relation familiale visant à attester ou contester une légitimité à revendiquer des droits sur un héritage ou sur une $\operatorname{dot}^{62}$. La mère des plaidoyers est, au pire, instrumentalisée en tant que prostituée ou étrangère pour disqualifier l'adversaire ${ }^{63}$ ; au mieux, et c'est le cas le plus fréquent, elle n'est que le maillon d'une chaine généalogique qui la relie à son fils. Car c'est bien de la relation mère-fils dont il est question, jamais, ou quasi jamais, de la relation mère-fille. Rien de surprenant à cela, la cité est un "club d'hommes ${ }^{64}$ ", et si depuis le décret de Périclès, l'Athénienne existe individuellement en tant que mère potentielle d'un citoyen athénien ${ }^{65}$, c'est toujours d'un fils dont il s'agit. La fille n'a de lien civique qu'avec son père, son frère ou un tuteur qui la donne en mariage et la dote. On connaît, cependant, deux plaidoyers de deux orateurs différents où les relations entre une mère et sa fille sont mises en scène. Dans le premier, que nous devons à Andocide (son discours Sur les mystères), les liens entre le couple mèrefille ne sont évoqués que furtivement au sein d'une affaire de mœurs particulièrement complexe. Le second, le Contre Nééra d'Apollodore, est entièrement consacré à une femme et cela est suffisamment rare pour que cela soit noté. L'action est bien intentée contre un homme, Stéphanos, mais la plaidoirie se porte contre sa femme, Nééra, et la fille de cette dernière, Phanô. Comme on le verra, c'est principalement autour de la question du mariage des filles et de son importance pour la communauté civique des Athéniens que se joue la relation fille/mère chez les orateurs. Un mariage qui n'intéresse les orateurs que dans la mesure où il pervertit les normes sociales de la cité. 


\section{Chrysilla, sa fille et sa petite-fille}

17 La première évocation des relations mère-fille dans la prose des orateurs se trouve dans un réquisitoire d'Andocide contre Callias rédigé en 399 av. J.-C. dans son plaidoyer Sur les Mystères. Cette histoire particulièrement sordide nous amène, comme le remarque très justement Jean-Baptiste Bonnard ${ }^{66}$, dans un imbroglio familial particulièrement complexe. Membre du genos des Kéryces, le riche et influent stratège de la guerre de Corinthe, Callias, fils d'Hipponicos et parent d'Andocide, était en charge du sacerdoce de dadouque de Déméter et Korè à Éleusis. Il épousa la fille d'Ischomachos, mais peu après son mariage, il accueillit sous son toit Chrysilla, la mère de cette épouse et la séduit. Trahie par sa mère et son mari, condamnée à vivre dans la même maison que le couple, la fille d'Ischomachos jugea qu'il était préférable de mourir plutôt que de vivre dans pareille honte. Après une tentative de suicide ratée, la fille d'Ischomachos, déshonorée, s'enfuit alors de la maison : la « mère avait chassé la fille ${ }^{67}$ ». Et lorsqu'il en eut assez de la mère, Callias la renvoya à son tour ${ }^{68}$. De l'union de Chrysilla avec Callias était né un fils pour lequel ce dernier revendiqua la fille épiclère d'Épilycos. Mais en réalité, il projetait de l'épouser lui-même. Or, celle-ci se trouvait être la fille que sa deuxième femme, la fille d'Ischomachos, avait eue avec Épilycos, et donc la petite-fille de Chrysilla ${ }^{69}$. Cette fille épiclère était également revendiquée par Andocide en tant que parent le plus proche: elle était la fille de son oncle maternel. Afin de déjouer les intentions d'Andocide, Callias qui occupait des fonctions sacerdotales lui intenta, par le biais d'un homme de paille, Képhisios, un procès pour impiété. Andocide aurait, pendant la cérémonie des Mystères, déposé dans l'Éleusinion un rameau de suppliant, ce qui est passible de la peine de mort. Andocide riposta en s'en prenant à la moralité de Callias et en dénonçant les conditions dans lesquelles était né son fils :

Eh bien citoyen, cherchez si en Grèce on vit pareille turpitude : un homme épousant une femme, puis la mère, et les ayant toutes deux à la fois, et la fille chassée par la mère (thugatritên mêtera). Et quand il vit avec celle-ci, il prétend épouser la fille ( thugatêr) d'Épilycos, afin que la grand-mère (têthê) soit chassée par la petite-fille ( thugatridê). Et son fils (to pais), quel nom lui donner? Personne je crois n'a de nom assez subtil pour le trouver. Car voici trois femmes avec lesquelles aura vécu son père (patêr): il est le fils (uios) de l'une, à ce qu'il dit, frère (adelphos) de l'autre, oncle (theios) de la troisième. Que peut-il donc bien être? Ædipe, ou Égisthe ? Ou comment, enfin, le nommer ? ${ }^{70}$

Concentrons-nous sur l'histoire conjugale de Callias telle qu'elle est décrite, à tort ou à raison, par Andocide. Celle-ci nous met en présence de trois générations de femmes en ligne directe, la fille épiclère, sa mère (la fille d'Ischomachos) et sa grand-mère (Chrysilla). Ces trois femmes, sont, au sein de l'oikos de Callias, en concurrence les unes avec les autres puisque deux d'entre elles, la fille d'Ischomachos et sa mère Chrysilla, ont partagé la couche du stratège, tandis que la troisième, si Callias parvient à revendiquer la fille épiclère, s'apprête à le faire. En insistant sur les termes de parenté qui lient les trois femmes les unes aux autres, le réquisitoire d'Andocide met en avant la question de l'adultère (Égisthe), celle de l'inceste (Ædipe) et surtout la confusion des rôles générationnels entre les femmes dans le mariage. Laissons de côté la question supposée de l'inceste du premier et du deuxième type qui a suscité une abondante littérature ${ }^{71}$. La question de l'adultère suffit à elle seule pour montrer la double anormalité de ces relations. En effet, il s'agit d'une relation hors mariage et, de surcroît, la mère a pris la place de sa fille tandis que la petite-fille pourrait prendre celle de sa grand-mère - et qui 
souligne la perversion des liens de transmission qui devrait unir une fille, sa mère et sa grand-mère. Andocide, qui revendique pour lui-même la petite-fille de Chrysilla, évoque la monstruosité de la stratégie matrimoniale de Callias dans le but évident de choquer les juges et de les rallier à sa cause. Mais il dénonce avec autant de vigueur les turpitudes de Callias que le comportement dévoyé de Chrysilla. Cette dernière, en ne respectant pas la place qui lui est assignée comme mère et grand-mère dans l'ordre des générations, a enfreint les normes de la cité, qui sont théâtralisées dans un registre tragique sur la scène athénienne.

\section{Nééra et Phanô}

19 Le Contre Nééra est un discours incontournable lorsqu'on s'intéresse à la condition de la femme athénienne et ses différents statuts, - courtisane, concubine et épouse légitime et Nééra est probablement la prostituée dont on connait le mieux la vie, vue par le regard d'un homme athénien ${ }^{72}$. Toutefois les historiens se sont plus intéressés aux thèmes de la prostitution et du mariage qu'à celui des relations entre Nééra et sa fille Phanô $\hat{o}^{73}$. Pourtant, en regardant de près le récit d'Apollodore, on s'aperçoit que la vie de la fille est traitée en miroir de celle de sa mère et que la transmission de l'une à l'autre se fait sur le mode de la répétition.

L'histoire a lieu dans un contexte tout aussi sordide que celle rapportée par Andocide. En 340 av. J.-C., Théomnestos, homme politique athénien, accuse Nééra d'avoir, en épousant Stéphanos, transgressé la loi qui interdit à une étrangère d'être l'épouse d'un athénien. La plaidoirie est prononcée par son gendre et beau-frère, Apollodore, fils de l'esclave affranchi Pasion et citoyen de fraîche date.

21 Après avoir racheté sa liberté avec l'aide de son amant Phrynion, la courtisane Nééra s'enfuit d'Athènes en emportant avec elle les biens que lui avait offerts son amant ainsi que les deux esclaves mises à son service, pour s'installer à Mégare. Elle y rencontre Stéphanos qui devient son amant régulier. Ce dernier parvient à la convaincre de venir habiter avec lui à Athènes avec la promesse d'un mariage et de la reconnaissance de ses trois enfants, deux garçons et une fille. Une fois installée à Athènes avec Stéphanos, Nééra, selon le plaideur, continua d'exercer avec l'accord de son époux, ses activités de courtisane, activité dont Stéphanos tirait profit, par chantage, en accusant d'adultère les hommes à qui elle se vendait ${ }^{74}$. En effet, une loi de Dracon permettait à tout homme de tuer le séducteur de son épouse, de sa mère, de sa sœur ou de sa concubine, surpris en flagrant délit ${ }^{75}$.

Apprenant que Nééra était à Athènes, son ancien amant Phrynion la revendique et accuse Stéphanos de recel des biens que Nééra avait emporté de chez lui. Pour éviter le procès les deux parties conclurent un arrangement stipulant que tous deux disposeraient de Nééra autant de jours l'un que l'autre ${ }^{76}$.

Nééra était venue à Athènes accompagnée de ses trois enfants en bas âge. On sait peu de chose sur les pères de ces derniers. Les avait-elle eus de différents amants ou Stéphanos était-il leur père ${ }^{77}$ ? Toujours est-il que Stéphanos les introduisit dans sa phratrie et dans son dème ${ }^{78}$ en les reconnaissant comme siens. Nous n'en saurons pas plus sur les deux fils de Nééra. En revanche, sa fille Phanô, accusée par Apollodore d'avoir été donnée illégalement en mariage à un Athénien alors qu'elle était étrangère, va faire l'objet d'une longue partie de la plaidoirie. On va le voir, la vie de Phanô n'est alors qu'une succession d'aventures répétant l'histoire de sa mère. 

trente mines en la faisant passer pour l'enfant qu'il aurait eue d'une première femme athénienne. Après un an de mariage, alors que Phanô était enceinte, Phrastor décida de la renvoyer chez son père sans restituer la dot. Deux raisons à ce renvoi : l'indocilité de Phanô et ses mauvaises habitudes de luxe héritées de sa mère ${ }^{79}$. Par ailleurs, Phrastor avait acquis la certitude que Phanô n'était pas la fille d'une Athénienne, mais celle de Nééra, donc d'une étrangère, et qu'en conséquence le mariage était illégal. Stéphanos dut renoncer à récupérer la dot de Phanô. Peu après la répudiation de Phanô, Phrastor tombe malade; brouillé avec sa famille, il fait appel à Nééra et Phanô pour le soigner. En échange, cette dernière lui demande de reconnaître le fils qu'elle a eu avec lui, né peu après leur séparation ${ }^{80}$. Si apparemment les membres de la phratrie acceptèrent d'introduire l'enfant, il n'en fut pas de même des membres de son genos, celui des Brytides. Ces derniers refusèrent l'admission du fils de Phrastor argumentant qu'il était de la fille de Nééra, et donc d'une étrangère ${ }^{81}$.

Entre-temps, Stéphanos, qui connaît des problèmes d'argent, utilise, pour en obtenir, le même procédé du chantage à l'adultère, mais cette fois en se servant non pas de Nééra, mais de sa fille. La jeune Phanô prend ici la place d'une mère sans doute vieillissante. Lors de la venue à Athènes d'Épainétos, un ancien amant de Nééra, Stéphanos attira celui-ci dans le lit de Phanô et le surprit en flagrant délit d'adultère. Il contraint alors Épainétos de lui verser trente mines de réparation pécuniaire. Ce dernier s'exécuta mais intenta aussitôt un procès à Stéphanos. Si Épainétos reconnut avoir eu des relations avec Phanô, il contesta l'accusation d'adultère, arguant du fait que Phanô n'était pas la fille de Stéphanos mais celle de Nééra. Il invoqua également la loi selon laquelle il n'y a pas d'adultère lorsque les relations ont eu lieu avec une prostituée dans une maison close ou avec une femme qui racole sur la voie publique ${ }^{82}$. En d'autres termes, Phanô était une prostituée, et la maison de Stéphanos, un bordel. Une conciliation entre les deux hommes permit d'éviter le procès. Épainétos versa mille drachmes pour constituer une dot à Phanô. En contrepartie, Phanô serait mise à disposition d'Épainétos toutes les fois qu'il séjournerait à Athènes et qu'il aurait le désir de la voir ${ }^{83}$. Ainsi, Phanô a-t-elle fait, comme sa mère, l'objet d'une conciliation qui la met à disposition d'un homme.

Disposant à nouveau d'une dot pour Phanô, Nééra et Stéphanos ${ }^{84}$ cherchèrent une fois de plus à marier Phanô pour en faire l'épouse d'un citoyen. La faisant passer pour une Athénienne, ils jetèrent leur dévolu sur Théogénes, un citoyen pauvre mais respectable ; celui-ci venait d'être désigné par tirage au sort pour être l'Archonte-roi. Phanô devint alors, basilinna, et dut, à ce titre, accomplir les rites secrets et sacrés pratiqués lors des Anthestéries. Elle présida, notamment, à ceux de l'union sacrée avec Dionysos ${ }^{85}$, rituel réservé à une Athénienne légitimement mariée et qui, de surcroît, devait être socialement vierge, parthenos, au moment de son mariage. Il va sans dire que Phanô, fille de la courtisane étrangère Nééra, ayant elle-même des mœurs légères, ne répondait en aucun cas à ces exigences. Démasquée par le Conseil de l'Aréopage, Phanô fut à nouveau répudiée par Théogénes. Ainsi, Nééra, cherchant à accomplir son telos de mère, n'a de cesse de vouloir marier sa fille à un citoyen athénien. Mais le récit du plaideur l'installe dans une position d'échec où elle ne parvient qu'à transmettre à sa fille sa propre condition, c'est-à-dire celle d'étrangère et de courtisane. Un échec qui est, selon le plaideur, une condition essentielle pour sauvegarder la vertu de toutes les filles des citoyens d'Athènes. En effet, si Nééra parvenait à ses fins, si au terme du procès, elle était acquittée, sa qualité d'épouse de citoyen reconnue, sa fille légitimée, les frontières entre 
la femme mariée et la courtisane seraient abolies et par effet de contamination, l'avenir des jeunes filles de la cité serait menacé :

Pensez aux femmes athéniennes, craignez que les filles des citoyens pauvres ne trouvent plus de maris. Aujourd'hui, même quand elles sont dans la gêne, la loi leur constitue une dot suffisante, pour peu que la nature ne les ait pas disgraciées. Mais que par votre acquittement, la loi soit tournée en dérision et cesse de commander, du coup, la prostitution s'étendra aux filles de citoyens, à toutes celles qui ne pourront être mariées faute d'argent. En revanche, les courtisanes (étairas) seront élevées à la dignité de femmes libres (éleuthérôn gunaikôn), quand elles auront le privilège d'avoir des enfants légitimes (paidopoieisthai) à leur gré et de participer aux mystères, aux sacrifices, aux prérogatives des citoyens ${ }^{86}$.

Le plaidoyer d'Apollodore est dirigé contre Stéphanos et son groupe politique, mais en préférant s'attaquer aux comportements et aux statuts de Nééra et de sa fille, le plaideur s'en prend au "maillon le plus faible ${ }^{87}$ » de la famille, les femmes. Il est, en effet, surprenant qu'à aucun moment, la légitimité des fils de Nééra ne soit mise en doute. Tout se passe comme si, dans le plaidoyer, pour l'orateur, la transmission des mères ne pouvait concerner que leurs filles, comme si, seules les femmes, la « race des femmes », pouvaient menacer l'unité de la société.

Nous avons vu, à travers les textes tragiques et la poésie d'un hymne homérique, que les mères revendiquent un rôle très actif dans le mariage de leur fille. Conduire leur fille jusqu'au mariage est pour les mères l'aboutissement de leur devoir de transmission. Ce sont elles qui les préparent à quitter le foyer familial et les assistent dans les rites qui préludent au transfert de la mariée de la maison de son père à celle de son époux. Il est par ailleurs vraisemblable que, dans les bonnes familles, les mères soient consultées par leur époux sur le choix du conjoint. Ainsi, en transmettant les fonctions d'épouse à leurs filles, les mères contribuent à leur intégration dans la cité. Et c'est précisément cette contribution des mères, parce qu'elle intéresse la communauté civique dans son ensemble, qui apparaît dans les plaidoyers des orateurs. Peu présentes dans les discours des orateurs, les relations entre les mères et leurs filles ne sont mentionnées que lorsqu'elles risquent de mettre en danger la cohésion de la cité athénienne ; une menace qui réside dans le rôle que jouent l'une et l'autre dans l'institution du mariage. Dans le réquisitoire d'Andocide contre Callias, le mariage de la fille d'Ischomachos avec Callias, puis celui de sa propre mère, Chrysilla, avec ce même Callias, et enfin la perspective du mariage de la petite fille de Chrysilla toujours avec Callias, dénaturent l'ordre des places de ces trois femmes dans la génération. Dans le second réquisitoire, la légitimité du mariage de la courtisane Nééra avec un citoyen athénien est contestée par Apollodore et, par voie de conséquence, ceux de sa fille Phanô le sont également. Et si, plaide Apollodore, les juges reconnaissent une légitimité au mariage de Nééra, c'est la légitimité et les mœurs de toutes les jeunes filles des citoyens athéniens qui seront mises en cause.

Si le mariage est un événement " privé » et familial, ces conséquences, rappelle Florence Gherchanoc $^{88}$, sont d'ordre public. En accompagnant leurs filles dans tous les rituels jusqu'à la demeure de leurs époux, les mères affirment leur rôle fondamental au sein des structures familiale et sociale de la cité - un rôle qui peut être alors la source d'un conflit affiché sur la place publique. 


\section{BIBLIOGRAPHIE}

Bodiou L., Brulé P. et Pierini L. (2005), « En Grèce antique, la douloureuse obligation de la maternité », Clio. HFS [En ligne] 21. URL : http://clio.revues.org/1441; DOI : 10.4000/clio.1441

Bodiou L. (2006), « De la mère à la fille : fusion, séparation, réconciliation. L'exemple de Déméter et Koré », dans Bodiou L., Frère D., Mehl V. dir., L'expression des corps. Gestes, attitude, regards dans l'iconographie antique, Rennes.

Bonnard J.-B. (2002), « Phèdre sans inceste. À propos de la théorie de l'inceste du deuxième type et de ses applications en histoire grecque », $R H 1, \mathrm{n}^{\circ} 621$, p. 77-107.

Bresson A. (1981), « Règles de nomination dans la Rhodes Antique », DHA 7, p. 345-362.

Bruit Zaidman L. (1991), « Les filles de Pandore. Femmes et rituels dans les cités », dans Schmitt Pantel P. dir., Histoire des femmes en Occident, I, Paris, p. 363-403.

- (1996), «Le temps des jeunes filles dans le cité grecque : Nausicaa, Phrasikleia, Timareta et les autres », Clio. HFS [En ligne] 4. URL : http://clio.revues.org/431; DOI : 10.4000/clio.431

- (2011), «Épouses et prostituées : le cas Nééra », dans Boehringer S., Sebillotte Cuchet V. éd., Hommes et femmes dans l'antiquité grecque et romaine. Le genre méthode et documents, Paris, p. 44-47.

- (2012), « Déméter Déesse et mère », dans Caiozzo A. et Ernoult N. dir., Femmes médiatrices et ambivalentes. Mythes et imaginaires, Paris, p. 381-391.

Buxton R. (1996), La Grèce et l'imaginaire. Les contextes de la mythologie, Paris.

Calame Cl. (1997), «L'Hymne homérique à Déméter comme offrande : regard rétrospectif sur quelques catégories de l'anthropologie de la religion grecque », Kernos 10, p. 111-133.

Carvalho De Silvia M. S. (1992), trad. Michel R., « Les mystères d'Éleusis », DHA 18, p. 93-135.

Cid Lopez R. M. (2009), Madres y maternidades. Construcciones culturales en la civilizacion clasica, Oviedo.

Davidson J. N. (1997), Courtesans and Fishcakes: The Consuming Passions of Classical Athens, Londres.

Frontisi-Ducroux F. (2003), « Idéaux féminins : le cas de la Grèce ancienne », Topique 82, p. 111-119.

Gherchanoc F. (1998), « Le lien filial dans l'Athènes classique : Pratiques et acteurs de sa reconnaissance ", Mètis XIII, p. 313-344.

- (2012), L'oikos en fête. Célébration familiales et sociabilité en Grèce ancienne, Paris.

Hamel D. (2003), Trying Neaira. The True Story of a Courtesan's Scandalous Life in Ancient Greece, New Haven-Londres.

Héritier F. (1994-1995), « L'inceste dans les textes de la Grèce classique et postclassique », Mètis IX-X, p. 99-115.

- (1994), Les deux sœurs et leur mère. Anthropologie de l'inceste, Paris.

Kahn-Lotard L. et Loraux N. (1981), « Mort. Les mythes grecs », dans Bonnefoy Y. éd., Dictionnaire des mythologies II, Paris, p. 117-124. 
Leduc Cl. (1991), « Comment la donner en mariage? », dans Duby G., Perrot P. et Schmitt Pantel P. dir., Histoire des femmes, L’Antiquité, Paris, p. 259-316.

- (1994), « Citoyenneté et parenté dans la cité des Athéniens », Mètis IX-X, p. 51-68.

Lissarrague F. (1996), « Regards sur le mariage grec », dans Cavalier O. dir., Silence et fureur. Les femmes et le mariage en Grèce. Les antiquités grecques du musée Calvet, Avignon, p. 414-433.

Loraux N. (1991), « Qu'est-ce qu'une déesse ? », dans Duby G., Perrot P. et Schmitt Pantel P. éd., Histoire des femmes, L'Antiquité, Paris, p. 31-62.

- (1990), Les Mères en deuil, Paris.

Marrou H. I. (1981), Histoire de l'éducation dans l'Antiquité. 1. Le monde grec, Paris, (1 ère éd. 1948).

Miner J. (2003), « Courtesan, Concubine, Whore: Apollodorus' Deliberate Use of Terms for Prostitutes », AJPh 124, p. 19-37.

Morizot Y. (2004), " Offrandes à Artémis pour une naissance. Autour du relief d'Archinos », dans Dasen V. éd., Naissance et petite enfance dans l'Antiquité, Fribourg-Gœttingue, p. 159-170.

Mossé Cl. (2003), « Nééra, la courtisane », dans Loraux N. éd., La Grèce au féminin, Paris, p. 215-246.

Nancy Cl. (1984), «Euripide et le parti des femmes », QUCC NS 17, 2, p. 111-136.

Norcia S. (2011), Les relations mère-fille en Grèce ancienne, mémoire de master 2 de l'université Denis Diderot-Paris 7.

Paradiso A. (1988), «L'agrégation du nouveau-né au foyer familial : les Amphidromies », DHA 14, p. 203-218.

Parisinou E. (2000), « Lighting the World of Women: Lamp and Torches in the Hands of Women in the Late Archaic and Classical Periods », G\&R, Second series 47, 1, p. 19-43.

Rudhardt J. (1962), « La reconnaissance de la paternité : sa nature, sa portée dans la société athénienne : sur un discours de Démosthène », MH 19, p. 34-64.

- (1978), « À propos de l'hymne homérique à Déméter », MH 35, fasc. 1, p. 1-17.

Vérilhac A.-M. (1978-1982), Paides Aoroi. Poésie funéraire I-II, Athènes.

- (1985), «L’image de la femme dans les épigrammes funéraires grecques », La femme dans le monde méditerranéen. I. Antiquité, Lyon, p. 85-112.

Vérilhac A.-M. et Vial Cl. (1998), Le mariage grec du vi siècle av. J.-C. à l'époque d'Auguste, Paris.

Vernier B. (1996), « Théorie de l'inceste et construction d'objet. Françoise Héritier, la Grèce antique et les Hittites », Annales. HSH, 51 e année, 1, p. 173-200.

Vial Cl. (1985), « La femme athénienne vue par les orateurs », dans Vérilhac A.-M. dir., La femme dans le monde méditerranéen. I. Antiquité, Lyon, p. 47-60.

- (1996), « Statut et subordination », dans Cavalier O. dir., Silence et fureur. Les femmes et le mariage en Grèce. Les antiquités grecques du musée Calvet, Avignon, p. 339-357.

Villacèque N. (2013), Spectateurs de paroles! Délibération démocratique et théâtre à Athènes à l'époque classique, Rennes.

- (2014), « Ta mère ! Insulte et généalogie à la tribune démocratique », Cahiers « Mondes anciens » [En ligne] 5. URL : http://mondesanciens.revues.org/1242 ; DOI : 10.4000/mondesanciens.1242

Vilatte S. (1992), « Déméter et l'institution matrimoniale : le refus du passage », RBph, tome 70 fasc. 1, p. 115-134. 


\section{NOTES}

1. Il faut toutefois signaler le récent mémoire de master 2 de Sophia Norcia sur « Les relations mère-fille en Grèce ancienne » (Norcia 2011).

2. Isée, III, 30 : «Ces mêmes oncles ont déposé que leur neveu les avait conviés à la cérémonie du dixième jour de la fille qui était donnée comme sienne, et qu'ils y avaient assisté. [Ils] ont attesté que le père avait donné à l'enfant le nom de sa grand-mère ». Cf. Paradiso 1988.

3. Cf. Rudhardt 1962, p. 34-64, Gherchanoc 1998, p. 313-344 et Bonnard 2003.

4. Sur les rites funéraires, cf. Vérilhac 1985, p. 8 : « C'est d'ordinaire un mari, un père, un fils ou un frère qui ensevelit la défunte et, même si exceptionnellement c'est une mère ou une fille, elle ne peut, dans une épitaphe, s'écarter de l'opinion commune, or l'opinion commune c'est celle des hommes puisqu'ils sont les seuls à pouvoir s'exprimer librement. »

5. Sur l'autorité du père concernant la dation en mariage, voir Claude Vial 1996, p. 339-357.

6. Frontisi-Ducroux 2003.

7. Xénophon, Économique, VII, 14.

8. Morizot 2004.

9. C'est l'hypothèse de Florence Gherchanoc (2012, p. 46). Elle cite à ce propos Euripide, Aigeus, 5. Médée demande à Thésée : "Quel nom t'a donné ta mère la dixième nuit après ta naissance ? » et Aristophane, Les Nuées, v. 59-72 où le poète met en scène une querelle entre un père et une mère à propos de l'attribution du nom à un fils qui venait de naître dans le contexte d'un mariage hypogamique.

10. C'est notamment le cas dans la Rhodes antique. Cf. Alain Bresson (1981, p. 345) : « La première née prend le nom de sa grand-mère maternelle, la seconde, celui de sa grand-mère paternelle, la troisième un nom du côté de sa mère, la quatrième un nom du côté de son père. » Voir également Isée, III, Sur la succession de Pyrrhos, 30.

11. Platon, Gorgias 527a ; République 377c ; Lysis 205d. La transmission des récits mythologiques et des contes aux enfants est l'apanage des femmes. Cf. Buxton 1996, p. 33-34.

12. Il faut toutefois relativiser le nombre de participantes à ces fêtes. Selon Louise Bruit Zaidman (1996, p. 41) : «Cette parade des jeunes générations est réservée aux filles des familles les plus prestigieuses et les plus riches de la cité, notamment à Athènes la démocratique, qui sait faire appel aux plus fortunés de ses citoyens pour assurer l'éclat de ses fêtes. Les filles des eugeneis, les "biens nés", remplissent ainsi une triple fonction : se montrer, en tant que "filles à marier", manifester le prestige de leur famille, contribuer à l'éclat que la cité sait donner à ses fêtes pour la plus grande gloire de ses dieux et pour la sienne propre. »

13. Bruit Zaidman 1996, p. 33.

14. Bodiou, Brulé et Pierini 2007, p. 39.

15. Selon une expression de Louise Bruit Zaidman 1996, p. 44. Cf. Euripide, Médée, v. 239-240.

16. Sur cette question, voir François Lissarrague (1996, p. 415) ainsi que l'étude d'Anne-Marie Vérilhac et Claude Vial (1998).

17. Sur Euripide et le monde des femmes, voir Vérilhac 1996. Il faut toutefois, à la suite de l'article de Claire Nancy (1984), nuancer le « féminisme » d'Euripide.

18. Euripide, Alceste, v. 316-320.

19. Euripide, Iphigénie à Aulis, v. 459.

20. Euripide, Iphigénie à Aulis, v. 728.

21. Euripide, Iphigénie à Aulis, v. 738-740.

22. Euripide, Iphigénie à Aulis, v. 963-964.

23. Euripide, Électre, v. 264 ; Oreste : « Ta mère, pour sa fille, a permis cet hymen? » 
24. Euripide, Iphigénie en Tauride, v. 818 : « Tu as reçu de ta mère l'eau du bain pour Aulis?» Cette scène est fréquemment reproduite sur les vases grecs. Cf. le lebes gamikos du peintre de Mikonos, vers 460 av. J.-C., Copenhague, Nationalmuseet, inv. 9165.

25. Voir les scènes de toilette nuptiale sur un épinétron à figures rouges, "toilette de la mariée Harmonie ", peintre d'Éretrie, vers 420 av. J.-C., Athènes, Musée national, inv. 1629, ainsi que sur une pyxis à figures rouges, "toilette de la mariée ", Sicile, groupe d'Adrano, vers 325 av. J.-C., Moscou, Musée Pouchkine, inv. 510.

26. Euripide, Les Troyennes, v. 314.

27. Euripide, Les Troyennes, v. 353-354 : «Ma mère, couronne mon front victorieux et réjouis-toi de mes noces royales. »

28. Euripide, Iphigénie à Aulis, v. 905 : «C'était pour toi que je l'avais couronnée, et je la conduisais ici pour être ta femme.»

29. Euripide, Iphigénie à Aulis, v. 722 : «Et moi, où offrirai-je le banquet pour les femmes? ». À ce propos Florence Gherchanoc (2012, p. 26) se demande si cette sociabilité «genrée » est propre aux besoins de la scène.

30. Euripide, Iphigénie à Aulis, v. 732.

31. Euripide, Iphigénie à Aulis, v. 691-694 : «Crois bien que j'éprouverai moi-même ces sentiments - je ne puis donc te les reprocher - quand j'escorterai ma fille aux sons de l'hyménée. »

32. Cf. Parisinou 2000, p.33. Voir la scène de mariage sur une loutrophore attique à figures rouges (450-425 av. J. C.), Boston, Museum of Fine Arts, inv. 03.802.

33. Euripide, Les Phéniciennes, v. 344-346 : «Je n'ai point allumé pour toi la flamme du feu, comme c'est la coutume des noces pour une mère bienheureuse. » Comme le remarque très justement Sophie Norcia (2011, p. 68) : «La transition symbolique du feu d'une mère à l'autre - la bellemère porte une torche lorsqu'elle conduit le jeune couple à leur chambre nuptiale - participe à l'intégration de la fille au sein de son nouvel oikos. »

34. Euripide, Ion, v. 1473-1474 :«Ion : Ce fils de vierge, hélas ! n'était qu'un bâtard. Créuse : Ah ! Ce n'est pas parmi les torches, ni les danses que mon hymen t'a enfanté. » Cf. Lissarrague 1996, p. 418 et Gherchanoc 2012, p. 133. Un mariage sans le rituel des torches est un mariage clandestin et les enfants sont considérés comme illégitimes.

35. Euripide, Iphigénie à Aulis, v. 690-695.

36. Euripide, Les Troyennes, v. 355.

37. Nancy 1984, p. 121-122.

38. Vérilhac 1996, p. 359.

39. Villacèque 2013, p. 88-91.

40. Anthologie palalatine, VII, 487. Voir également Anthologie palatine, VII, 649: «Au lieu des douceurs de la couche nuptiale et des graves chants d'hyménée, ta mère a dressé sur ce tombeau de marbre une jeune vierge qui a ta taille et ta beauté. »

41. Cf. Vérilhac 1978-1982, p. 156-160.

42. Hymne homérique à Déméter, v. 78 .

43. Bruit Zaidman 2012.

44. Sur le passage de nom de Korè à celui de Persphone voir Bruit Zaidman 2012.

45. Sur la symbolique de la grenade, voir Vérilhac et Vial 1998, p. 340-341.

46. Bruit Zaidman 2012, p. 382.

47. Cf. Calame 1997, p. 129 : «Pour formuler en des termes encore plus généraux les fonctions conjuguées de Déméter et de Coré dans leur domaine d'action commun, on constatera que le récit, en se focalisant sur la relation entre la mère et la fille auxquelles cèdent successivement les représentants du pouvoir "patriarcal" que sont Hadès et Zeus, place tout le processus civilisateur de l'agriculture sous la protection des femmes alors que le sacrifice sanglant est essentiellement affaire d'hommes. » 
48. Calame 1997, p. 125. Voir également Sylvie Vilatte (1992, p.130) : «D'ailleurs le mariage d'Hadès et Coré met fin au cycle de perte de la fille, cycle qui se produit à chaque génération ; car le surprenant retour de Perséphone auprès de sa mère, retour qui prend à rebours la réalité de la vie matrimoniale, s'accompagne, au plan des générations, d'une stérilité du couple HadèsPerséphone. »

49. Il est donc difficile de suivre Lydie Bodiou (2006, p. 315) lorsqu'elle affirme : " Pour Déméter, il s'agit à la fois de renoncer à la toute puissance maternelle et à la fin des plaisirs d'Aphrodite. Pour Korè, si la séparation est aussi douloureuse, elle est aussi le début d'une histoire, celle de sa vie de femme. " Sur l'oscilliation de Korè-Perséphone entre le jeune fille et le jeune femme, voir Kahn-Lyotard et Loraux 1981, p. 122.

50. Parmi une très longue bibliographie, on retiendra: Rudhardt 1978, Carvalho 1992, Vilatte 1992, Calame 1997, Bodiou 2006, Bruit Zaidman 2012 et 2013.

51. Loraux 1991, p. 40.

52. Sur cette question, voir Jérôme Wilgaux (2011, p. 342) : «À l'époque classique, les sources littéraires démontrent d'ailleurs que les hommes peuvent se revendiquer tout autant de leur ascendance maternelle que de leur ascendance paternelle, et que les identités et pratiques religieuses se transmettent de manière générale par les hommes comme par les femmes. »

53. Vial 1985, p. 52.

54. Démosthène, XLVII, Contre Évergos et Mnésiboulos, 57 ; XXVII, Contre Aphobos I, 17-18; XXX, Contre Onètor I, 9-10.

55. Démosthène, XLVI, Contre Stéphanos II.

56. Démosthène, XXI, Contre Midias,79; XLII, Contre Phénippos 27 : « Ma mère à moi est en vie, elle habite dans ma maison où elle a apporté une dot [...]. »

57. C'est le cas de la mère de Démosthène; cf. Démosthène, XXVII, Contre Aphobos I.

58. Lysias, XXXI, Contre Philon, 22.

59. Démosthène, XIX, Sur les forfaitures de l'ambassade 199: «Ces juges ne savait-ils pas tout d'abord que tu lisais les livres de ta mère pendant ses initiations et que, dans ton enfance, tu te vautrais dans des thiases d'ivrognes?»

60. Démosthène, XVIII, Sur la couronne, 129 : «Je n'ai pas à me demander ce que je dois dire de toi et des tiens, mais je me demande ce que je rappellerai en premier lieu. Sera-ce que ton père Tromès était esclave d'Elpis qui enseignait l'écriture près du Théseion? Il portait de grosses entraves et un carcan. Ou bien ta mère, grâce à ses mariages [gamoi] en plein jour dans la boutique voisine du héros Calamitès, a élevé le beau portrait et l'excellent acteur que tu es? »

61. Lysias, XXXI, Contre Philon, 20-22.

62. Sur le statut juridique de la mère, voir l'étude d'Aurélie Damet dans la présente publication.

63. Comme l'a montré Noémie Villacèque (2014) dans une récente étude sur l'insulte dans les tribunaux démocratiques, la mère de l'adversaire est devenue pour les orateurs une «cible de choix, car l'invectiver permettait de mettre en doute la citoyenneté de son fils et de le faire ainsi sortir de la communauté civique ».

64. Selon une expression d'Henri Irénée Marrou (1948, p. 64) de l'édition de 1981.

65. Leduc 1994, p. 60.

66. Cf. Bonnard 2002.

67. Andocide, I, Sur les Mystères, 125.

68. Idem.

69. Cf. Bonnard 2002. L'auteur publie à la fin de son article un stemma des différents mariages de Callias très utile pour nous permettre d'y voir plus clair dans cet imbroglio familial.

70. Andocide, I, Sur les Mystères, 128.

71. Sur cette question de l'inceste, voir le débat entre Françoise Héritier (1994-1995 et 1994), Bernard Vernier (1996) et Jean-Baptiste Bonnard (2002).

72. Voir les biographies que lui ont consacrées Debra Hamel (2003) et Claude Mossé (2003). 
73. Voir cependant les remarques judicieuses mais brèves de Louise Bruit Zaidman (2011) et Claude Mossé (2003).

74. [Démosthène], LIX, Contre Nééra, 41.

75. Démosthène, XXIII, Contre Aristocratès, 53 et Lysias, I, Sur le meurtre d'Ératosthène, 31.

76. [Démosthène], LIX, Contre Nééra, 47.

77. À ce sujet, cf. Mossé 2003, p. 225.

78. [Démosthène], LIX, Contre Nééra, 13.

79. [Démosthène], LIX, Contre Nééra, 50.

80. [Démosthène], LIX, Contre Nééra, 56-57.

81. [Démosthène], LIX, Contre Nééra, 61.

82. [Démosthène], LIX, Contre Nééra, 67.

83. [Démosthène], LIX, Contre Nééra, 7. Le texte de l'accord ne précise pas sa durée mais, dans la mesure où Phanô s'est remariée après la conciliation, on peut penser que l'accord n'a pas été appliqué ou, comme le suggère Claude Mossé (2003, p. 233), que l'accusation d'Apollodore est une pure invention.

84. Nééra est fortement impliquée dans la décision de marier sa fille puisque le texte du plaidoyer précise bien que cette démarche est à l'initiative du couple, Stéphanos et Nééra.

85. [Démosthène], LIX, Contre Nééra, 73 : «Elle a accompli, au nom de la cité, les rites traditionnels à l'égard des dieux, des rites nombreux, sacrés et indicibles (aporrêta). »

86. [Démosthène], LIX, Contre Nééra, 113.

87. Cf. Bruit Zaidman 2011, p. 46.

88. Gherchanoc 2012, p. 23.

\section{RÉSUMÉS}

Les textes tragiques et l'Hymne homérique à Déméter ainsi que les épitaphes funéraires, montrent combien la préparation au mariage est un moment important, voire essentiel, de la relation mère/fille. Conduire leurs filles jusqu'au mariage est, pour les mères, l'aboutissement de leur devoir de transmission. C'est ainsi qu'elles contribuent à leur intégration dans la cité. En revanche, chez les orateurs attiques, la question des relations mères/filles est peu évoquée, si ce n'est dans le plaidoyer Sur les Mystères d'Andocide et dans le discours Contre Nééra d'Apollodore (attribué à Démosthène), car dans ces circonstances, ce que les mères transmettent à leurs filles menace l'unité de la cité.

Tragic textes and the Homeric Hymn to Demeter, as funerary, epitaphs, show how the marriage's preparation is an important moment, even essential, in mother / daughters relationship. Drive their daughters until marriage is, for mothers, the culmination of their duty of transmission. Thereby they contribute to their integration in the city. By contrast, in the attics orators speeches, mother / daughter relationship is rarely mentioned, except in Andocides' speech On the Mysteries, and in Apollodorus' speech Against Neaera (cf. Demosthenes), because in these cases, what the mothers pass to their daughters threatens the unity of the city. 
INDEX

Mots-clés : mariage, mère, fille, transmission, théâtre, Euripide, orateur attique, Démosthène, Andocide, épitaphe

Keywords : marriage, mother, daughter, transmission, theater, Euripides, attic orator, Demosthenes, Andocides, epitaph

\section{AUTEUR}

NATHALIE ERNOULT

Centre Pompidou, Université Paris Diderot-Paris 7, ANHIMA - UMR8210 